Pacific

Journal of

Mathematics

\title{
QUASICONFORMAL CONJUGACY CLASSES \\ OF PARABOLIC ISOMETRIES \\ OF COMPLEX HYPERBOLIC SPACE
}

YOUNGJU KIM 


\title{
QUASICONFORMAL CONJUGACY CLASSES OF PARABOLIC ISOMETRIES OF COMPLEX HYPERBOLIC SPACE
}

\author{
YOUNGJU KIM
}

\begin{abstract}
We investigate the quasiconformal conjugacy classes of parabolic isometries acting on complex hyperbolic space. Our main result is that a screw parabolic isometry cannot be quasiconformally conjugate to a translation. This implies that a cyclic group generated by a screw parabolic isometry is not quasiconformally stable in its deformation space.
\end{abstract}

We are interested in the quasiconformal deformation theory of a complex hyperbolic quasi-Fuchsian group. We mainly focus on the case that the group is a cyclic group generated by a parabolic isometry.

We recall the definition of quasiconformal stability from Kleinian group theory (see, for instance, [Bers 1970; Kapovich 2008; Marden 1974; Maskit 1988]). Let $\Gamma$ be a finitely generated discrete subgroup of the orientation-preserving isometry group Isom $\left(\mathbb{H}^{n+1}\right)$ acting on real hyperbolic $(n+1)$-space $\mathbb{H}^{n+1}$. Such a group $\Gamma$ is called a Kleinian group. A representation $\rho: \Gamma \rightarrow \operatorname{Isom}\left(\mathbb{H}^{n+1}\right)$ is said to be a deformation if it is a discrete, faithful and type-preserving representation. The Kleinian group $\Gamma$ is said to be quasiconformally stable if any deformation $\rho: \Gamma \rightarrow \operatorname{Isom}\left(\mathbb{H}^{n+1}\right)$ sufficiently near the identity deformation is obtained by a quasiconformal conjugation. That is, there is a quasiconformal mapping of the boundary at infinity, $\phi: \partial \mathbb{M}^{n+1} \rightarrow \partial \mathbb{W}^{n+1}$, such that $\rho(g)=\phi \circ g \circ \phi^{-1}$ for any $g \in \Gamma$.

In $\mathbb{M}^{2}$ and $\mathbb{M}^{3}$, a geometrically finite Kleinian group is quasiconformally stable [Bers 1970; Marden 1974]. This is one of the fundamental results in the deformation theory of Kleinian groups. However, there is a nonelementary geometrically finite Kleinian group of hyperbolic 4-space which is not quasiconformally stable [Kim 2011]. This is mainly due to the presence of screw parabolic isometries in hyperbolic 4-space.

This research was supported by Basic Science Research Program through the National Research Foundation of Korea (NRF) funded by the Ministry of Education, Science and Technology (NRF2011-0021240).

MSC2010: primary 20H10, 30C65; secondary 51M10, 22E40, $32 \mathrm{G} 07$.

Keywords: Heisenberg group, parabolic isometry, complex hyperbolic space, quasiconformal stability. 
Hyperbolic $(n+1)$-space $\mathbb{H}^{n+1}$ has the natural boundary at infinity $\hat{\mathbb{R}}^{n}$. Every isometry of $\mathbb{M}^{n+1}$ extends continuously to a Möbius transformation of $\hat{\mathbb{R}}^{n}$ which is a finite composition of reflections in codimension-1 spheres or hyperplanes, and vice versa. On the boundary at infinity $\hat{\mathbb{R}}^{n}$, a parabolic isometry is Möbius conjugate to $x \mapsto A x+e_{1}$ with $A \in \mathrm{SO}(n), A\left(e_{1}\right)=e_{1}$, where $e_{1}=(1,0, \ldots, 0) \in \mathbb{R}^{n}$. If $A=I$, then it is called a strictly parabolic isometry or a translation; otherwise it is a screw parabolic isometry. There are no screw parabolic isometries if $n<3$. This means that there is only one conformal, and hence quasiconformal, conjugacy class of parabolic isometries in lower dimensions. In $\mathbb{H}^{4}$, screw parabolic isometries are not quasiconformally conjugate to translations. Furthermore, there are infinitely many distinct quasiconformal conjugacy classes of screw parabolic isometries. Let $\Gamma$ be a cyclic group generated by a translation. Then we can deform $\Gamma$ into a cyclic group $\Gamma^{\prime}$ generated by a screw parabolic isometry such that $\Gamma$ is arbitrary close to $\Gamma^{\prime}$. Hence, the cyclic group $\Gamma$ is not quasiconformally stable in its deformation space. We can generalize this to a nonelementary Kleinian group of $\mathbb{M}^{4}$ (see [Kim 2011] for details). On the other hand, it is known that a convex cocompact (i.e., geometrically finite without parabolic isometries) Kleinian group is quasiconformally stable in any dimension [Izeki 2000].

Now, we consider the case of complex hyperbolic space $\mathbb{U}_{\mathbb{C}}^{2}$. A complex hyperbolic quasi-Fuchsian group is a discrete, faithful, type-preserving and geometrically finite representation of the fundamental group of a surface in the group $\mathrm{PU}(2,1)$ of holomorphic isometries acting on complex hyperbolic space $\mathbb{T}_{\mathbb{C}}^{2}$ [Goldman 1999; Parker and Platis 2010; Schwartz 2007]. It is the complex counterpart of a Kleinian group of real hyperbolic space. The deformation space is the set of all such groups factored by the conjugation action of the holomorphic isometry group $\mathrm{PU}(2,1)$. Naturally, we can ask if a complex hyperbolic quasi-Fuchsian group is quasiconformally stable in its deformation space (see [Parker and Platis 2010] for more related questions). To that end, we consider a cyclic group generated by a parabolic isometry of $\mathbb{Q}_{\mathbb{C}}^{2}$.

The boundary at infinity of complex hyperbolic space can be identified with the one-point compactification of the Heisenberg group $\mathscr{H}: \partial \mathbb{W}_{\mathbb{C}}^{2}=\mathscr{H} \cup\{\infty\}$. A holomorphic isometry of $\mathbb{T}_{\mathbb{C}}^{2}$ extends continuously to an extended Heisenberg group automorphism of $\partial \mathbb{U}_{\mathbb{C}}^{2}$, and vice versa. On $\partial \mathbb{U}_{\mathbb{C}}^{2}$, a parabolic isometry of $\mathbb{U}_{\mathbb{C}}^{2}$ is conjugate to either a Heisenberg translation or the composition of a vertical translation and a rotation by an element of $\mathrm{PU}(2,1)$. We call the latter a screw parabolic isometry.

A Heisenberg translation can be conjugate to either a horizontal translation or a vertical translation by an element of $\mathrm{PU}(2,1)$. We can conjugate a horizontal translation (or a vertical translation) further by an element of $\operatorname{PU}(2,1)$ so that the translation length is 1 with respect to the Cygan norm of the Heisenberg group. 
Therefore, we have the following classification of conformal classes of parabolic isometries up to the conjugation action of $\mathrm{PU}(2,1)$ :

- a horizontal translation $T_{(1,0)}$,

(1) - a vertical translation $T_{(0,1)}$,

- a 1-parameter family of screw parabolic isometries $\left\{A_{\theta}: \theta \in(0,2 \pi)\right\}$,

where

$$
T_{(\zeta, \nu)}=\left(\begin{array}{ccc}
1 & -\sqrt{2} \bar{\zeta} & -|\zeta|^{2}+i \nu \\
0 & 1 & \sqrt{2} \zeta \\
0 & 0 & 1
\end{array}\right) \in \operatorname{SU}(2,1)
$$

for $\zeta \in \mathbb{C}, v \in \mathbb{R}$, and

$$
A_{\theta}=\left(\begin{array}{ccc}
1 & 0 & i \\
0 & e^{i \theta} & 0 \\
0 & 0 & 1
\end{array}\right) \in \mathrm{SU}(2,1)
$$

for $\theta \in(0,2 \pi)$ (see Section 1B for details).

Miner [1994] showed that a horizontal translation and a vertical translation are not quasiconformally conjugate. That is, no quasiconformal mapping of the Heisenberg group conjugates a horizontal translation to a vertical one. We prove here that a screw parabolic isometry is not quasiconformally conjugate to a translation, as follows:

Theorem 3.3. Let $T_{(0,1)}(z, t)=(z, t+1)$ be a vertical translation and $A(z, t)=$ $\left(e^{i \theta} z, t+1\right)$, for $\theta \in(0,2 \pi)$, be a screw parabolic automorphism of the Heisenberg group $\mathcal{H}$. Then $A$ is not quasiconformally conjugate to $T_{(0,1)}$.

Theorem 3.7. Let $T_{(1,0)}(z, t)=(z+1, t+2 \operatorname{Im} \bar{z})$ be a horizontal translation and $A_{\theta}(z, t)=\left(e^{i \theta} z, t+1\right)$, for $\theta \in(0,2 \pi)$, be a screw parabolic automorphism of the Heisenberg group $\mathcal{H}$. Then $A_{\theta}$ is not quasiconformally conjugate to $T_{(1,0)}$.

A screw parabolic isometry is called rational if some iteration of it becomes a translation; otherwise, it is called irrational. For a rational screw parabolic isometry $A$, the $\operatorname{order}$ of $A$ is the smallest positive integer $n$ such that $A^{n}$ becomes a translation. For the 1-parameter family of screw parabolic isometries from (1), we prove that a rational screw parabolic isometry cannot be quasiconformally conjugate to an irrational screw parabolic isometry in Corollary 3.4, that two distinct rational screw parabolic isometries are quasiconformally conjugate only if they have the same order in Corollary 3.5, and that two distinct irrational screw parabolic isometries are not quasiconformally conjugate to each other in Proposition 3.6. In summary, together with the result of [ibid.], we have the following distinct quasiconformal conjugacy classes of parabolic isometries of $\mathbb{U}_{\mathbb{C}}^{2}$ (compare with the list (1)): 
- a horizontal translation $T_{(1,0)}$;

- a vertical translation $T_{(0,1)}$;

- a subfamily of irrational screw parabolic isometries $\left\{A_{\vartheta}: \vartheta \in(0,2 \pi)\right.$ irrational $\}$;

- a subfamily of rational screw parabolic isometries $\left\{A_{2 \pi i / n}: n=2,3, \ldots\right\}$.

Let $\Gamma<\mathrm{PU}(2,1)$ be a cyclic group generated by a vertical translation. Then we can deform $\Gamma$ into a cyclic group $\Gamma^{\prime}$ generated by a screw parabolic isometry such that $\Gamma$ is arbitrary close to $\Gamma^{\prime}$ with respect to the $l^{2}$ norm of $\mathrm{PU}(2,1)$. Applying Theorem 3.3, this shows that $\Gamma$ is not quasiconformally stable in its deformation space. Thus, we have:

Theorem. Let $\Gamma<\mathrm{PU}(2,1)$ be a cyclic group generated by a vertical translation. Then it is not quasiconformally stable in its deformation space.

This paper is organized as follows. In Section 1, we recall some basic facts related to complex hyperbolic geometry, the Heisenberg group and the theory of quasiconformal mappings. In Section 2, we construct a family of horizontal curves in a cylindrical region and compute the modulus of the curve family. This curve family will be used to prove Theorem 3.3 in Section 3. We will also prove Theorem 3.7 in Section 3.

\section{Preliminaries}

Throughout this section, we use [Goldman 1999] as references for the basic definitions of complex hyperbolic geometry and [Korányi and Reimann 1985; 1995] for the theory of quasiconformal mappings.

1A. Complex hyperbolic space. Let $\mathbb{C}^{2,1}$ be the complex vector space $\mathbb{C}^{3}$ with the Hermitian form of signature $(2,1)$ given by

$$
\langle\boldsymbol{z}, \boldsymbol{w}\rangle=\boldsymbol{w}^{*} J \boldsymbol{z}=z_{1} \bar{w}_{3}+z_{2} \bar{w}_{2}+z_{3} \bar{w}_{1},
$$

where the Hermitian matrix is

$$
J=\left(\begin{array}{lll}
0 & 0 & 1 \\
0 & 1 & 0 \\
1 & 0 & 0
\end{array}\right) .
$$

Consider the following subspaces of $\mathbb{C}^{2,1}$ :

$$
\begin{gathered}
V_{-}=\left\{z \in \mathbb{C}^{2,1}:\langle z, z\rangle<0\right\}, \\
V_{0}=\left\{z \in \mathbb{C}^{2,1}-\{0\}:\langle z, z\rangle=0\right\} .
\end{gathered}
$$

Let $\mathbb{P}: \mathbb{C}^{2,1}-\{0\} \rightarrow \mathbb{C P}^{2}$ be the canonical projection onto complex projective space. Then complex hyperbolic space $\mathbb{H}_{\mathbb{C}}^{2}$ is defined to be $\mathbb{P} V_{-}$and the boundary at 
infinity $\partial \mathbb{Q}_{\mathbb{C}}^{2}$ to be $\mathbb{P} V_{0}$. We define the Siegel domain model of complex hyperbolic space by considering the section defined by $z_{3}=1$. For any $z=\left(z_{1}, z_{2}\right) \in \mathbb{C}^{2}$, we lift the point $z$ to $z=\left(z_{1}, z_{2}, 1\right) \in \mathbb{C}^{2,1}$, called the standard lift of $z$. Then $\langle\boldsymbol{z}, \boldsymbol{z}\rangle=z_{1}+z_{2} \bar{z}_{2}+\bar{z}_{1}$. Hence the Siegel domain model of complex hyperbolic space is defined by

$$
\mathbb{H}_{\mathbb{C}}^{2}=\left\{\left(z_{1}, z_{2}\right) \in \mathbb{C}^{2}: 2 \operatorname{Re}\left(z_{1}\right)+\left|z_{2}\right|^{2}<0\right\} .
$$

The boundary is the one-point compactification of the paraboloid defined by $\left\{\left(z_{1}, z_{2}\right) \in \mathbb{C}^{2}: 2 \operatorname{Re}\left(z_{1}\right)+\left|z_{2}\right|^{2}=0\right\}$. The standard lift of $\infty$ is $(1,0,0) \in \mathbb{C}^{2,1}$.

The Bergman metric $\rho$ on $\mathbb{W}_{\mathbb{C}}^{2}$ is defined by

$$
\cosh ^{2}\left(\frac{\rho(z, w)}{2}\right)=\frac{\langle\boldsymbol{z}, \boldsymbol{w}\rangle\langle\boldsymbol{w}, \boldsymbol{z}\rangle}{\langle\boldsymbol{z}, \boldsymbol{z}\rangle\langle\boldsymbol{w}, \boldsymbol{w}\rangle}
$$

where $z$ and $w$ are the standard lifts of $z$ and $w \in \mathbb{U}_{\mathbb{C}}^{2}$. Let $\operatorname{SU}(2,1)$ be the group of unitary matrices which preserve the given Hermitian form with determinant 1 . Then the group of holomorphic isometries of $\mathbb{H}_{\mathbb{C}}^{2}$ is $\mathrm{PU}(2,1)=\mathrm{SU}(2,1) /\left\{I, \omega I, \omega^{2} I\right\}$, where $\omega=(-1+i \sqrt{3}) / 2$ is a cube root of unity.

Let $z=\left(z_{1}, z_{2}\right) \in \partial \mathbb{Q}_{\mathbb{C}}^{2}$ be a finite point with standard lift $z=\left(z_{1}, z_{2}, 1\right)$ satisfying

$$
2 \operatorname{Re}\left(z_{1}\right)+\left|z_{2}\right|^{2}=0 .
$$

We write $\zeta=z_{2} / \sqrt{2} \in \mathbb{C}$. Then (8) implies that $2 \operatorname{Re}\left(z_{1}\right)=-2|\zeta|^{2}$. We can also write $z_{1}=-|\zeta|^{2}+i v$ for $v \in \mathbb{R}$. Thus,

$$
z=\left(\begin{array}{c}
-|\zeta|^{2}+i \nu \\
\sqrt{2} \zeta \\
1
\end{array}\right)
$$

for $\zeta \in \mathbb{C}$ and $v \in \mathbb{R}$. Thus, we identify the boundary $\partial \mathbb{R}_{\mathbb{C}}^{2}$ with the one-point compactification of $\mathbb{C} \times \mathbb{R}$. Furthermore, an element $T_{(\zeta, v)} \in \mathrm{SU}(2,1)$ of (2) is the unique unipotent upper triangular matrix which sends $(0,0) \in \mathbb{C} \times \mathbb{R}$ to the finite point $(\zeta, v) \in \mathbb{C} \times \mathbb{R}$. The group structure of the unipotent upper triangular matrices induces a group multiplication on $\mathbb{C} \times \mathbb{R}$, which is the Heisenberg group structure.

1B. Heisenberg group. The Heisenberg group $\mathscr{H}$ can be described as the set of pairs $(z, t) \in \mathbb{C} \times \mathbb{R}$ with the group multiplication

$$
\left(z_{1}, t_{1}\right) \cdot\left(z_{2}, t_{2}\right)=\left(z_{1}+z_{2}, t_{1}+t_{2}+2 \operatorname{Im} z_{1} \bar{z}_{2}\right) .
$$

The Cygan norm on $\mathscr{H}$ is defined by $|(z, t)|=\left(|z|^{4}+t^{2}\right)^{1 / 4}$, and the Cygan metric $d$ is given by

$$
d\left(\left(z_{1}, t_{1}\right),\left(z_{2}, t_{2}\right)\right)=\left|\left(z_{1}, t_{1}\right)^{-1} \cdot\left(z_{2}, t_{2}\right)\right| .
$$


The Heisenberg group $\mathscr{H}$ acts on itself by left translation: $T_{\left(z_{0}, t_{0}\right)}(z, t)=\left(z_{0}, t_{0}\right) \cdot(z, t)$ for $\left(z_{0}, t_{0}\right) \in \mathscr{H}$. A Heisenberg translation of the form $T_{(0, t)}$ for $t \in \mathbb{R}$ is called a vertical translation. The unitary group $U(1)$ acts by rotations: $(z, t) \mapsto(\lambda z, t)$ for a unit $\lambda \in \mathbb{C}-\{1\}$. Real dilation is defined by $(z, t) \mapsto\left(r z, r^{2} t\right)$ for $r \in \mathbb{R}_{+}-\{1\}$. A parabolic Heisenberg group automorphism is either a Heisenberg translation or the composition of a vertical translation and a rotation. We call the latter type screw parabolic. A screw parabolic automorphism $A(z, t)=\left(e^{i \theta} z, t+s\right)$, for $\theta \in(0,2 \pi), s \in \mathbb{R}$, is said to be rational if some iteration of it becomes a Heisenberg translation. Otherwise, it is said to be irrational. The Heisenberg similarity group is generated by Heisenberg translations, rotations, and real dilations.

It is known to many people that there are two conformal conjugacy classes of Heisenberg translations. More precisely, we can conjugate a Heisenberg translation by a holomorphic isometry of $\mathbb{H}_{\mathbb{C}}^{2}$ to obtain a horizontal translation or a vertical translation in the following way. Let $T$ be a nonvertical translation. We may conjugate $T$ by a Heisenberg automorphism $m(z, t)=\left(\lambda e^{i \theta} z, \lambda^{2} t\right)$ for $\lambda \in \mathbb{R}_{+}$, $\theta \in[0,2 \pi)$, such that

$$
m \circ T \circ m^{-1}=T_{(r, s)},
$$

where $T_{(r, s)}(z, t)=(z+r, t+s+2 r \operatorname{Im} \bar{z})$ for some real numbers $r$ and $s$ with $r \neq 0$. For a computation, we note that for $w \in \mathbb{C},(w, t)(r, s)(-w,-t)=(r, s+4 r \operatorname{Im} w)$ and hence $s+4 r \operatorname{Im} w=0$ if $\operatorname{Im} w=-s / 4 r$. We conjugate both sides of (12) by a Heisenberg translation $T_{(w, t)}$ with $\operatorname{Im} w=-s / 4 r$ as follows:

$$
T_{(w, t)} m \operatorname{Tm}^{-1} T_{(w, t)}^{-1}=T_{(w, t)} T_{(r, s)} T_{(w, t)}^{-1}=T_{(r, 0)} .
$$

Conjugating both sides of (13) by a dilation $L(z, t)=\left(L z, L^{2} t\right)$ for some $L \in \mathbb{R}_{+}$, we have

$$
L T_{(w, t)} m T m^{-1} T_{(w, t)}^{-1} L^{-1}=L T_{(r, 0)} L^{-1}=T_{(1,0)},
$$

where $T_{(1,0)}(z, t)=(z+1, t+2 \operatorname{Im} \bar{z})$. Thus, any nonvertical translation $T$ is conjugate to $T_{(1,0)}$ by a Heisenberg automorphism.

A screw parabolic isometry can be conjugated to $A_{\theta}(z, t)=\left(e^{i \theta} z, t+1\right)$, with $\theta \in(0,2 \pi)$, by an element of $\mathrm{SU}(2,1)$. In addition, two distinct normalized screw parabolic isometries are not $\mathrm{SU}(2,1)$-conjugate to each other. Therefore, we have the following classification of conformal classes of parabolic isometries up to the conjugation action of the holomorphic isometries of $\mathbb{Q}_{\mathbb{C}}^{2}$ :

- a horizontal translation $T_{(1,0)}(z, t)=(z+1, t+2 \operatorname{Im} \bar{z})$;

- a vertical translation $T_{(0,1)}(z, t)=(z, t+1)$; 
- a 1-parameter family of screw parabolic isometries

$$
\left\{A_{\theta}(z, t)=\left(e^{i \theta} z, t+1\right): \theta \in(0,2 \pi)\right\} .
$$

1C. Quasiconformal mappings. Let $\phi: \mathscr{H} \rightarrow \mathscr{H}$ be a homeomorphism. We define

$$
M(p, r)=\sup _{\{q: d(p, q)=r\}} d(\phi p, \phi q) \quad \text { and } \quad m(p, r)=\inf _{\{q: d(p, q)=r\}} d(\phi p, \phi q)
$$

for $p \in \mathscr{H}$ and $r>0$.

Definition 1.1. A homeomorphism $\phi: \mathscr{H} \rightarrow \mathscr{H}$ is called $K$-quasiconformal if the function

$$
H(p)=\limsup _{r \rightarrow 0} \frac{M(p, r)}{m(p, r)}
$$

for $p \in \mathscr{H}$ is uniformly bounded by $K$.

We also need to use the Carnot-Carathéodory metric $d_{\mathrm{cc}}$ for our proof with quasiconformal mappings. A smooth curve $\gamma:[0,1] \rightarrow \mathscr{H}$ is horizontal if, for all $t \in[0,1]$, its tangent vector $\dot{\gamma}(t)$ lies in the subspace of the tangent space spanned by the vector fields $X=\partial / \partial x+2 y \partial / \partial t$ and $Y=\partial / \partial y-2 x \partial / \partial t$ for $(x, y, t) \in \mathbb{C} \times \mathbb{R}$. We define a quadratic form $g$ on the planes generated by vector fields $X$ and $Y$ such that $X$ and $Y$ are orthonormal. Then the Carnot-Carathéodory length of $\gamma$ is given by

$$
l(\gamma)=\int_{0}^{1} g(\dot{\gamma}(t), \dot{\gamma}(t))^{1 / 2} d t
$$

and the Carnot-Carathéodory distance $d_{\mathrm{cc}}$ between two points $p, q \in \mathscr{H}$ is the infimum of the Carnot-Carathéodory lengths of all horizontal curves connecting $p$ to $q$.

Let $\Gamma$ be a family of piecewise- $C^{1}$ horizontal curves. Denote by $\Sigma_{\Gamma}$ the collection of nonnegative Borel measurable functions $\sigma: \mathscr{H} \rightarrow \mathbb{R}$ such that $\int_{\gamma} \sigma \geq 1$ for all $\gamma \in \Gamma$. These are the so-called admissible functions. Then we define the modulus of $\Gamma$ by

$$
\mathrm{M}(\Gamma)=\inf _{\sigma \in \Sigma_{\Gamma}} \int_{\mathscr{H}} \sigma^{4} d \mathrm{vol} .
$$

We now relate the modulus of a curve family to a quasiconformal mapping.

Theorem 1.2 [Korányi and Reimann 1995]. If a homeomorphism $\phi: \mathscr{H} \rightarrow \mathscr{H}$ is $K$-quasiconformal, then

$$
\frac{1}{K^{2}} \mathrm{M}(\Gamma) \leq \mathrm{M}(\phi \Gamma) \leq K^{2} \mathrm{M}(\Gamma)
$$

for any curve family $\Gamma$. 
The Cygan metric $d$ and the Carnot-Carathéodory metric $d_{\mathrm{cc}}$ give us the same classes of quasiconformal mappings since they are bi-Lipschitz related:

Theorem 1.3 [Basmajian and Miner 1998]. For any $p, q \in \mathscr{H}$,

$$
d(p, q) \leq d_{\mathrm{cc}}(p, q) \leq \sqrt{\pi} d(p, q) .
$$

Finally, we need the following property of quasiconformal mappings.

Proposition 1.4 [Korányi and Reimann 1995]. There exists a constant $C$ such that for any $K$-quasiconformal mapping $\phi: \mathscr{H} \rightarrow \mathscr{H}$,

$$
\frac{M(p, r)}{m(p, r)} \leq e^{K C}
$$

for any $p \in \mathscr{H}$ and $r>0$.

\section{The modulus of a cylinder}

We construct here a family of piecewise smooth horizontal curves in a cylindrical region and compute its modulus. Let $\alpha_{0}:[0,1] \rightarrow \mathscr{H}$ be a piecewise smooth horizontal curve defined by $\alpha_{0}(t)=\alpha^{1}(t) * \alpha^{2}(t) * \alpha^{3}(t) * \alpha^{4}(t)$ (see Figure 1), where

$$
\begin{array}{rlrl}
\alpha^{1}(t) & =(2 t i, 0), & & 0 \leq t \leq \frac{1}{4}, \\
\alpha^{2}(t) & =\left(2 t-\frac{1}{2}+\frac{1}{2} i, 2 t-\frac{1}{2}\right), & & \frac{1}{4} \leq t \leq \frac{1}{2}, \\
\alpha^{3}(t) & =\left(\frac{1}{2}+\left(\frac{3}{2}-2 t\right) i, 2 t-\frac{1}{2}\right), & \frac{1}{2} \leq t \leq \frac{3}{4}, \\
\alpha^{4}(t)=(2-2 t, 1), & \frac{3}{4} \leq t \leq 1 .
\end{array}
$$

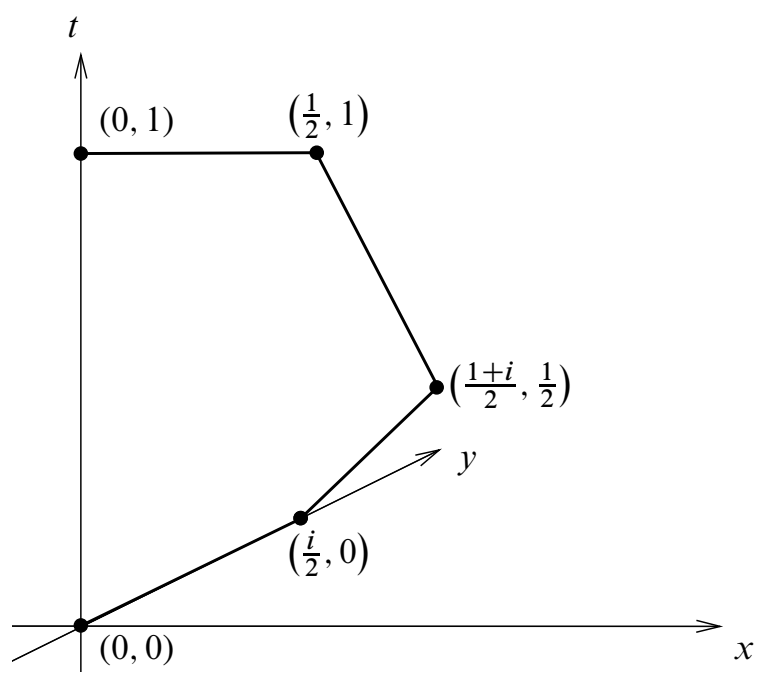

Figure 1. A piecewise smooth horizontal curve $\alpha_{0}(t)$. 
Note that $\alpha_{0}(0)=(0,0), \alpha_{0}(1)=(0,1)$, and $\left|\dot{\alpha}^{i}(t)\right|=2$. The Carnot-Carathéodory length of $\alpha_{0}$ is

$$
l\left(\alpha_{0}\right)=\sum_{i=1}^{4} \int_{0}^{1 / 4}\left|\dot{\alpha}^{i}(t)\right| d t=2 .
$$

Translating $\alpha_{0}$ by $T_{(z, 0)}$, for $(z, 0) \in \mathscr{H}$, produces a piecewise smooth horizontal curve $\alpha_{z}$, given by

$$
\alpha_{z}(t)=T_{(z, 0)} \alpha_{0}(t), \quad 0 \leq t \leq 1,
$$

from $\alpha_{z}(0)=(z, 0)$ to $\alpha_{z}(1)=(z, 1)$. Let $\alpha_{z}^{i}(t)=T_{(z, 0)} \alpha^{i}(t)$. Then $\alpha_{z}(t)=$ $\alpha_{z}^{1}(t) * \alpha_{z}^{2}(t) * \alpha_{z}^{3}(t) * \alpha_{z}^{4}(t)$, where

$$
\begin{array}{ll}
\alpha_{z}^{1}(t)=(x, y+2 t,-4 x t), & 0 \leq t \leq \frac{1}{4}, \\
\alpha_{z}^{2}(t)=\left(x+2 t-\frac{1}{2}, y+\frac{1}{2}, 2 t-\frac{1}{2}-x+4 t y-y\right), & \frac{1}{4} \leq t \leq \frac{1}{2}, \\
\alpha_{z}^{3}(t)=\left(x+\frac{1}{2}, y+\frac{3}{2}-2 t, 2 t-\frac{1}{2}-3 x+4 t x+y\right), & \frac{1}{2} \leq t \leq \frac{3}{4}, \\
\alpha_{z}^{4}(t)=(x+2-2 t, y, 1+2(2-2 t) y), & \frac{3}{4} \leq t \leq 1 .
\end{array}
$$

Since Heisenberg translations are isometries with respect to the Carnot-Carathéodory metric, all curves $\alpha_{z}$ have Carnot-Carathéodory length 2. Define a family of curves $\Gamma_{r, R}$ for $0<r<R$ by

$$
\Gamma_{r, R}=\left\{\alpha_{z}: r \leq|z| \leq R\right\} .
$$

This family of curves defines a mapping $\alpha$ from the cylindrical region

$$
D=\left\{(x, y) \in \mathbb{C}: r^{2}<x^{2}+y^{2}<R^{2}\right\} \times[0,1]
$$

to $\mathscr{H}$, given by

$$
\alpha(x, y, t)=\alpha_{x+y i}(t) .
$$

Let $D_{i}=\left\{(x, y): r^{2}<x^{2}+y^{2}<R^{2}\right\} \times[(i-1) / 4, i / 4], i=1,2,3,4$, so that $D=\bigcup_{i=1}^{4} D_{i}$. Then the Jacobian determinant of $\alpha$ is given by

$$
|\mathrm{J} \alpha(x, y, t)|= \begin{cases}4|x| & \text { on } D_{1}, \\ 4|1+y| & \text { on } D_{2}, \\ 4|1+x| & \text { on } D_{3}, \\ 4|y| & \text { on } D_{4} .\end{cases}
$$

Lemma 2.1. For $1<r<R$, we have the following lower bound for the modulus of the curve family:

$$
\mathrm{M}\left(\Gamma_{r, R}\right) \geq \frac{1}{256}\left(R^{2}-r^{2}\right)\left(\frac{\pi}{2}-2 \arctan \frac{1}{\sqrt{r^{2}-1}}\right) .
$$


Proof. Let $\sigma$ be an arbitrary admissible function in $\Sigma_{\Gamma_{\sqrt{r}, \sqrt{R}}}$. By Hölder's inequality,

$$
1 \leq \int_{\alpha_{z}} \sigma \leq\left(\int_{0}^{1} \sigma^{2}\left(\alpha_{z}(t)\right) d t\right)^{1 / 2}\left(\int_{0}^{1}\left|\dot{\alpha}_{z}(t)\right|^{2} d t\right)^{1 / 2} .
$$

Since $\int_{0}^{1}\left|\dot{\alpha}_{z}(t)\right|^{2} d t=2$,

$$
\frac{1}{2} \leq \int_{0}^{1} \sigma^{2}\left(\alpha_{z}(t)\right) d t=\sum_{i=1}^{4} \int_{\frac{i-1}{4}}^{\frac{i}{4}} \sigma^{2}\left(\alpha_{z}^{i}(t)\right) d t .
$$

Applying Hölder's inequality to each term of the right-hand side, we have

$$
\begin{aligned}
\int_{\frac{i-1}{4}}^{\frac{i}{4}} \sigma^{2}\left(\alpha_{z}^{i}(t)\right)|\mathrm{J} \alpha|^{1 / 2} \frac{1}{|\mathrm{~J} \alpha|^{1 / 2}} & d t \\
\leq & \leq\left(\int_{\frac{i-1}{4}}^{\frac{i}{4}} \sigma^{4}\left(\alpha_{z}^{i}(t)\right)|\mathrm{J} \alpha| d t\right)^{1 / 2}\left(\int_{\frac{i-1}{4}}^{\frac{i}{4}} \frac{1}{|\mathrm{~J} \alpha|} d t\right)^{1 / 2} .
\end{aligned}
$$

From (30), using the Jacobian determinant (28) and (31), we have

$$
\begin{aligned}
\frac{1}{2} & \leq \sum_{i=1}^{4}\left(\int_{\frac{i-1}{4}}^{\frac{i}{4}} \sigma^{4}\left(\alpha_{z}^{i}(t)\right)|\mathrm{J} \alpha| d t\right)^{\frac{1}{2}}\left(\int_{\frac{i-1}{4}}^{\frac{i}{4}} \frac{1}{|\mathrm{~J} \alpha|} d t\right)^{\frac{1}{2}} \\
& \leq\left(\sum_{i=1}^{4}\left(\int_{\frac{i-1}{4}}^{\frac{i}{4}} \sigma^{4}\left(\alpha_{z}^{i}(t)\right)|\mathrm{J} \alpha| d t\right)^{\frac{1}{2}}\right)\left(\sum_{i=1}^{4}\left(\int_{\frac{i-1}{4}}^{\frac{i}{4}} \frac{1}{|\mathrm{~J} \alpha|} d t\right)^{\frac{1}{2}}\right) \\
& \leq \frac{1}{4}\left(\sum_{i=1}^{4}\left(\int_{\frac{i-1}{4}}^{\frac{i}{4}} \sigma^{4}\left(\alpha_{z}^{i}(t)\right)|\mathrm{J} \alpha| d t\right)^{\frac{1}{2}}\right)\left(\frac{1}{\sqrt{|x|}}+\frac{1}{\sqrt{|x+1|}}+\frac{1}{\sqrt{|y|}}+\frac{1}{\sqrt{|y+1|}}\right)
\end{aligned}
$$

Thus,

$$
\begin{aligned}
& 2\left(\frac{1}{\sqrt{|x|}}+\frac{1}{\sqrt{|x+1|}}+\frac{1}{\sqrt{|y|}}+\frac{1}{\sqrt{|y+1|}}\right)^{-1} \\
& \quad \leq \sum_{i=1}^{4}\left(\int_{\frac{i-1}{4}}^{\frac{i}{4}} \sigma^{4}\left(\alpha_{z}^{i}(t)\right)|\mathrm{J} \alpha| d t\right)^{1 / 2} \leq 4\left(\sum_{i=1}^{4} \int_{\frac{i-1}{4}}^{\frac{i}{4}} \sigma^{4}\left(\alpha_{z}^{i}(t)\right)|\mathrm{J} \alpha| d t\right)^{1 / 2}
\end{aligned}
$$

Equations (31), (32) and (33) only hold if $|\mathrm{J} \alpha| \neq 0$. However, when we estimate a lower bound of the modulus in (35), we will restrict the domain of the integration so that we may assume $|\mathrm{J} \alpha| \neq 0$.

Using the trivial inequality

$$
4 \int_{\alpha(D)} \sigma^{4} d \mathrm{vol} \geq \sum_{i=1}^{4} \int_{\alpha\left(D_{i}\right)} \sigma^{4} d \mathrm{vol}
$$


and defining $U=\left\{x, y: r^{2} \leq x^{2}+y^{2} \leq R^{2}, x \geq 1, y \geq 1\right\}$, we have

$$
\begin{aligned}
\int_{\mathscr{H}} \sigma^{4} d \mathrm{vol} & \geq \int_{\alpha(D)} \sigma^{4} d \mathrm{vol}=\frac{1}{4} \sum_{i=1}^{4} \int_{\alpha\left(D_{i}\right)} \sigma^{4} d \mathrm{vol} \\
& \geq \sum_{i=1}^{4} \iint_{U} \int_{\frac{i-1}{4}}^{\frac{i}{4}} \sigma^{4}\left(\alpha_{z}^{i}(t)\right)|\mathrm{J} \alpha| d t d x d y \\
& \geq \iint_{U} \frac{1}{4}\left(\frac{1}{\sqrt{|x|}}+\frac{1}{\sqrt{|x+1|}}+\frac{1}{\sqrt{|y|}}+\frac{1}{\sqrt{|y+1|}}\right)^{-2} d x d y \\
& \geq \frac{1}{256} \operatorname{Area}(U) ;
\end{aligned}
$$

the third inequality follows from (33) and the fact that $d \mathrm{vol}=4 d x d y d t$; for the last inequality we argue as follows:

$$
\begin{aligned}
& \left(\frac{1}{\sqrt{|x|}}+\frac{1}{\sqrt{|x+1|}}+\frac{1}{\sqrt{|y|}}+\frac{1}{\sqrt{|y+1|}}\right)^{-2} \\
& \geq\left(\frac{\sqrt{\mid x(x+1) y(y+1)}}{\sqrt{|(x+1) y(y+1)|}+\sqrt{|x y(y+1)|}+\sqrt{|x(x+1)(y+1)|}+\sqrt{|x(x+1) y|}}\right)^{2} \\
& \geq\left(\frac{\sqrt{|x(x+1) y(y+1)|}}{4 \sqrt{|(x+1) y(y+1)|+|x y(y+1)|+|x(x+1)(y+1)|+|x(x+1) y|}}\right)^{2} \\
& \geq \frac{1}{16} \cdot \frac{|x(x+1) y(y+1)|}{|(x+1) y(y+1)|+|x y(y+1)|+|x(x+1)(y+1)|+|x(x+1) y|} \\
& \geq \frac{1}{16} \cdot \frac{x(x+1) y(y+1)}{4 x(x+1) y(y+1)}=\frac{1}{64} \quad \text { if } x \geq 1, y \geq 1 .
\end{aligned}
$$

Since $\sigma$ was arbitrary, we obtain (see Figure 2)

$$
\mathrm{M}\left(\Gamma_{r, R}\right) \geq \frac{1}{256}\left(R^{2}-r^{2}\right)\left(\frac{\pi}{2}-2 \arctan \frac{1}{\sqrt{r^{2}-1}}\right)
$$

\section{Parabolic quasiconformal conjugacy classes}

Throughout this section, let $A(z, t)=A_{\theta}(z, t)=\left(e^{i \theta} z, t+1\right)$ be a screw parabolic automorphism of the Heisenberg group $\mathscr{H}$ for $\theta \in(0,2 \pi)$, and

$$
T_{\left(z_{0}, t_{0}\right)}(z, t)=\left(z+z_{0}, t+t_{0}+2 \operatorname{Im} z_{0} \bar{z}\right)
$$

be a Heisenberg translation for $\left(z_{0}, t_{0}\right) \in \mathscr{H}$. An injective map $\phi: \mathscr{H} \rightarrow \mathscr{H}$ is called quasisymmetric if there is a homeomorphism $\eta:[0, \infty) \rightarrow[0, \infty)$ such that

$$
d(x, y) \leq t d(x, z) \Longrightarrow d(\phi x, \phi y) \leq \eta(t) d(\phi x, \phi z)
$$

for $x, y, z \in \mathscr{H}, t \in[0, \infty)$. 


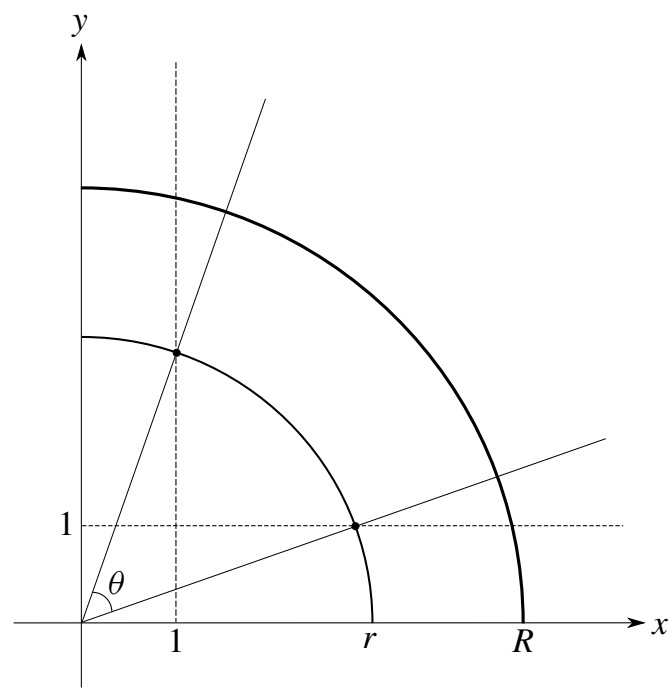

Figure 2. $\theta=\frac{\pi}{2}-2 \arctan \frac{1}{\sqrt{r^{2}-1}}$ (see previous page).

Theorem 3.1 [Heinonen and Holopainen 1997, Theorem 6.21]. If $\phi: \mathscr{H} \rightarrow \mathscr{H}$ is quasiconformal, then it is quasisymmetric.

Lemma 3.2. Let $\phi: \mathscr{H} \rightarrow \mathscr{H}$ be a quasiconformal map that fixes all integer points $(0, n)$ on the vertical axis. Then there exist a nondecreasing function $c:[0, \infty) \rightarrow$ $[0, \infty)$ and a constant $r_{0}>0$ satisfying:

- $\lim _{r \rightarrow \infty} c(r)=\infty$,

- for any $r e^{i \theta} \in \mathbb{C}$ with $r>r_{0}$,

$$
\left|p\left(\phi\left(r e^{i \theta}, 0\right)\right)\right| \geq c(r)
$$

where $p: \mathscr{H} \rightarrow \mathbb{C}$ is the vertical projection.

Proof. Throughout the proof, $[x]$ denotes the greatest integer less than or equal to $x$ for any $x \in \mathbb{R}$ and $B(p, r)$ the ball of radius $r \geq 0$ centered at $p \in \mathscr{H}$.

We use the property that the quasiconformal map $\phi$ is quasisymmetric for a homeomorphism $\eta:[0, \infty) \rightarrow[0, \infty)$ (Theorem 3.1). For any $r e^{i \theta} \in \mathbb{C}, r>0$,

$$
\frac{d\left((0,0),\left(0,[r]^{2}\right)\right)}{d\left((0,0),\left(r e^{i \theta}, 0\right)\right)}=\frac{[r]}{r} \leq 1
$$

implies that

$$
\frac{d\left(\phi(0,0), \phi\left(0,[r]^{2}\right)\right)}{d\left(\phi(0,0), \phi\left(r e^{i \theta}, 0\right)\right)}=\frac{[r]}{d\left((0,0), \phi\left(r e^{i \theta}, 0\right)\right)} \leq \eta(1) .
$$


Thus we have

$$
\frac{[r]}{\eta(1)} \leq d\left((0,0), \phi\left(r e^{i \theta}, 0\right)\right),
$$

and hence $\phi\left(r e^{i \theta}, 0\right)$ lies in the complement of the ball $B((0,0),[r] / \eta(1))$.

Similarly, for any $r e^{i \theta} \in \mathbb{C}$ and any integer $n$,

$$
\frac{d((0, n),(0,0))}{d\left((0, n),\left(r e^{i \theta}, 0\right)\right)}=\frac{\sqrt{|n|}}{\left(r^{4}+n^{2}\right)^{1 / 4}} \leq 1
$$

implies

$$
\frac{d(\phi(0, n), \phi(0,0))}{d\left(\phi(0, n), \phi\left(r e^{i \theta}, 0\right)\right)}=\frac{\sqrt{|n|}}{d\left((0, n), \phi\left(r e^{i \theta}, 0\right)\right)} \leq \eta(1) .
$$

Thus,

$$
\frac{\sqrt{|n|}}{\eta(1)} \leq d\left((0, n), \phi\left(r e^{i \theta}, 0\right)\right)
$$

and hence $\phi\left(r e^{i \theta}, 0\right)$ lies in the complement of the ball $B((0, n), \sqrt{|n|} / \eta(1))$. Since the integer $n$ was arbitrary, the image $\phi\left(r e^{i \theta}, 0\right)$ also lies in the complement of the set

$$
\bigcup_{n \in \mathbb{Z}} B\left((0, n), \frac{\sqrt{|n|}}{\eta(1)}\right) .
$$

Therefore, together with $(38)$, the image $\phi\left(r e^{i \theta}, 0\right)$ should lie in the complement of

$$
D_{r}=B\left((0,0), \frac{[r]}{\eta(1)}\right) \cup \bigcup_{n \in \mathbb{Z}} B\left((0, n), \frac{\sqrt{|n|}}{\eta(1)}\right) .
$$

Note that the $t$-intersects of the sphere of radius $[r] / \eta(1)$ centered at $(0,0)$ are $\pm\left(0,[r]^{2} / \eta^{2}(1)\right)$. We put

$$
n_{r}=\left[\frac{[r]^{2}}{\eta^{2}(1)}\right] \in \mathbb{N} .
$$

Take a positive real number $r_{0}$ large enough that $n_{r_{0}}>\eta(1)$.

To finish the proof, we will show that for $r>r_{0}, D_{r}$ contains an infinite cylinder

$$
C_{r}=\{(z, t) \in \mathscr{H}:|z| \leq c(r), t \in \mathbb{R}\},
$$

where $c(r)$ is a positive function such that $\lim _{r \rightarrow \infty} c(r)=\infty$. Since $D_{r}$ is symmetric with respect to the $z$-plane of $\mathscr{H}$, it suffices to show that the upper half of $D_{r}$, denoted by $\frac{1}{2} D_{r}$, contains a half cylinder $\frac{1}{2} C_{r}=\{(z, t) \in \mathscr{H}:|z| \leq c(r), t \geq 0\}$.

Since

$$
B\left((0, n), \frac{\sqrt{n_{r}}}{\eta(1)}\right) \subseteq B\left((0, n), \frac{\sqrt{n}}{\eta(1)}\right)
$$




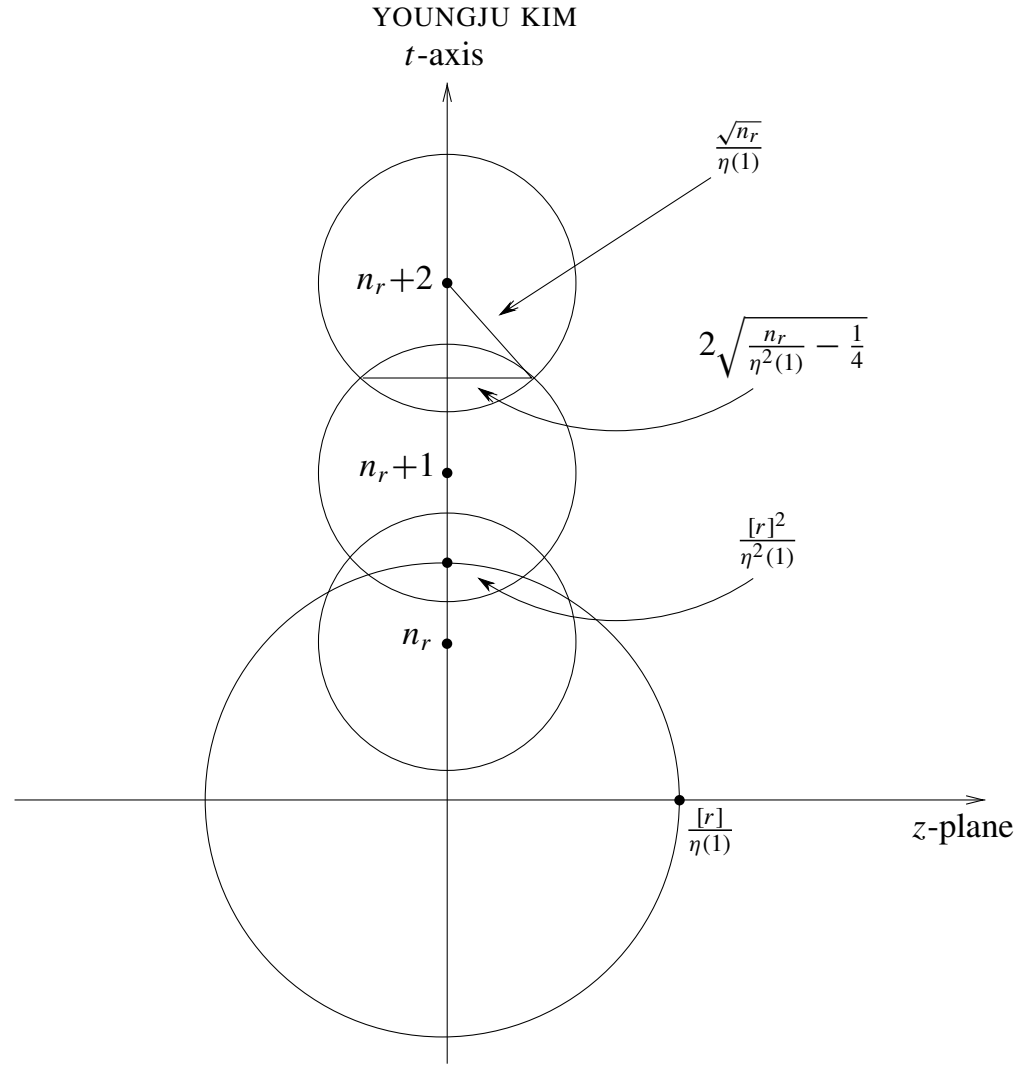

Figure 3. A set $\frac{1}{2} D_{r}^{\prime}$.

for $n>n_{r}>0$, the set $\frac{1}{2} D_{r}$ contains a proper subset $\frac{1}{2} D_{r}^{\prime}$ (see Figure 3):

$$
\frac{1}{2} D_{r}^{\prime}=B\left((0,0), \frac{[r]}{\eta(1)}\right) \cup \bigcup_{n \geq n_{r}} B\left((0, n), \frac{\sqrt{n_{r}}}{\eta(1)}\right) .
$$

Take

$$
c(r)=\min \left\{\sqrt{\frac{n_{r}}{\eta^{2}(1)}-\frac{1}{4}}, \frac{[r]}{\eta(1)}\right\} .
$$

Then we see that $\frac{1}{2} D_{r}^{\prime}$ contains the half cylinder $\frac{1}{2} C_{r}=\{(z, t) \in \mathscr{H}:|z| \leq c(r), t \geq 0\}$. Therefore, we have the lemma.

Theorem 3.3. Let $T_{(0,1)}(z, t)=(z, t+1)$ be a vertical translation and $A(z, t)=$ $\left(e^{i \theta} z, t+1\right)$, for $\theta \in(0,2 \pi)$, be a screw parabolic automorphism of the Heisenberg group $\mathcal{H}$. Then $A$ is not quasiconformally conjugate to $T_{(0,1)}$.

Proof. Suppose, to the contrary, that a $K$-quasiconformal map $\phi: \mathscr{H} \rightarrow \mathscr{H}$ exists such that

$$
\phi \circ A \circ \phi^{-1}=T_{(0,1)} .
$$


Let $\Gamma_{1}$ and $\Gamma_{2}$ be the cyclic groups generated by $A$ and $T_{(0,1)}$, respectively. Then $\phi$ projects to a $K$-quasiconformal mapping, called $\phi$ again, between the quotients. That is,

$$
\phi: \mathscr{H} / \Gamma_{1} \rightarrow \mathscr{H} / \Gamma_{2}
$$

If $\phi$ does not fix $(0,0)$, we compose (42) with a Heisenberg translation $m$ that sends $\phi(0,0)$ to $(0,0)$, so that we have

$$
m \circ \phi \circ A=m \circ T_{(0,1)} \circ \phi .
$$

Because the vertical translation $T_{(0,1)}$ commutes with all Heisenberg translations,

$$
m \circ \phi \circ A=T_{(0,1)} \circ m \circ \phi,
$$

and since $m$ is conformal, $m \circ \phi$ is also $K$-quasiconformal and fixes $(0,0)$. Hence, without loss of generality we may assume that the quasiconformal mapping $\phi$ of (42) fixes $(0,0)$.

Evaluating (42) at $(0,0)$ shows that $\phi(0,1)=(0,1)$. By induction, $\phi$ fixes all integer points $\{(0, n)\}$ on the vertical axes. The global estimate of Proposition 1.4 implies that there exists a constant $c_{0}$ such that for any given integer $r$, there is some $r^{\prime}$ for which

$$
B_{r^{\prime}} \subseteq \phi\left(B_{\sqrt{r}}\right) \subseteq B_{c_{0} r^{\prime}},
$$

where $B_{t}$ is a ball of radius $t$ centered at the origin. Since the integer point $(0, r)$ is fixed by $\phi$, the point $(0, r)$ also lies in $\phi\left(S_{\sqrt{r}}\right)$, where $S_{t}$ is the sphere of radius $t$ centered at the origin. Hence, (46) implies that

$$
r^{\prime} \leq \sqrt{r} \leq c_{0} r^{\prime}
$$

We consider the curve family $\Gamma_{\sqrt{r}, \sqrt{R}}$ from (26), where $r$ and $R$ are square integers satisfying $r_{0}<\sqrt{r}<\sqrt{R}$ and $r_{0}$ is the constant from Lemma 3.2. We put $\Gamma=\Gamma_{\sqrt{r}, \sqrt{R}}$ during this proof. All curves $\alpha_{z}$ in $\Gamma$ have length $\sqrt{\pi}$ and are homotopic to the generator of $\pi_{1}\left(\mathscr{H} / \Gamma_{1}\right)$.

We now compute the modulus of the family $\phi \Gamma$ consisting of the images of curves in $\Gamma$ under $\phi$. For any $r>0$, let $l_{r}$ denote the Carnot-Carathéodory distance from $(r, 0)$ to $A(r, 0)=\left(e^{i \theta} r, 1\right)$. Since the Carnot-Carathéodory distance is larger than or equal to the Cygan distance (Theorem 1.3), we have

$$
l_{r} \geq d\left((r, 0),\left(r e^{i \theta}, 1\right)\right)=\left(2^{4} r^{4} \sin ^{4} \frac{\theta}{2}+1\right)^{1 / 4} .
$$

Since the Carnot-Carathéodory distance and the Cygan distance are invariant under Heisenberg translations, the length of any horizontal curve from $(z, t)$ to $A(z, t)=\left(e^{i \theta} z, t+1\right)$ is at least $l_{|z|}$. Note that $\phi \Gamma$ is the family of curves connecting 
$\phi(p)$ to $A \phi(p)$, where $p$ belongs to the annulus $\{(z, 0) \in \mathscr{H}: \sqrt{r} \leq|z| \leq \sqrt{R}\}$. Using Lemma 3.2, we see that every curve $\gamma \in \phi \Gamma$ has length at least $l_{c(\sqrt{r})}$, where $c:[0, \infty) \rightarrow[0, \infty)$ is the function from the lemma.

We denote by $D$ the support of the curve family $\phi \Gamma$ :

$$
D=\phi \circ \alpha(\{z \in \mathbb{C}: \sqrt{r}<|z|<\sqrt{R}\} \times[0,1]),
$$

where $\alpha$ is the mapping of (27). Since each curve of $\Gamma$ is contained in a fundamental domain for the action of the cyclic group $\langle A\rangle$, and the quasiconformal homeomorphism $\phi$ conjugates $A$ to $T$ (see (42)), $D$ is also contained in a fundamental domain for the action of the cyclic group $\langle T\rangle$. Note that $T$ is the vertical translation by 1 . Thus, the intersection of a vertical line with $D$ might have several components, but the total length is bounded by 1 .

Now, let $\sigma=1 / l_{c(\sqrt{r})}$ be a constant function whose support is $D$. Then for any $\gamma \in \phi \Gamma$,

$$
\int_{\gamma} \sigma=\frac{1}{l_{c(\sqrt{r})}} l(\gamma) \geq 1
$$

and hence $\sigma$ is an admissible function of $\phi \Gamma$. Therefore,

$$
\mathrm{M}(\phi \Gamma) \leq \int_{\mathscr{H}} \sigma^{4} d \mathrm{vol}=\int_{D} \sigma^{4} d \mathrm{vol} \leq \sigma^{4} \int_{p(D)} 1 d x d y
$$

where $p: \mathscr{H} \rightarrow \mathbb{C}$ is the vertical projection.

Since the curves in $\Gamma$ belong to the ball $B_{\sqrt{R+1}}, D \subseteq \phi\left(B_{\sqrt{R+1}}\right)$. Again, Proposition 1.4 implies that

$$
B_{\widetilde{R}} \subseteq \phi\left(B_{\sqrt{R+1}}\right) \subseteq B_{c_{0}} \widetilde{R}
$$

for some $\widetilde{R}>0$. Because the integer point $(0, R+1)$ is fixed by $\phi,(0, R+1)$ lies in the image of the sphere $\phi\left(S_{\sqrt{R+1}}\right)$ and hence $\widetilde{R} \leq \sqrt{R+1} \leq c_{0} \widetilde{R}$. In particular, we have $c_{0} \widetilde{R} \leq c_{0} \sqrt{R+1}$. Therefore, we have $p(D) \subseteq p\left(B_{c_{0} \sqrt{R+1}}\right)$. From (50),

$$
\begin{aligned}
\sigma^{4} \int_{p(D)} 1 d x d y & \leq \frac{1}{l_{c(\sqrt{r})}^{4}} \int_{p\left(B_{c_{0} \sqrt{R+1}}\right)} 1 d x d y \\
& =\frac{\pi c_{0}^{2}(R+1)}{l_{c(\sqrt{r})}^{4}} \leq \frac{\pi c_{0}^{2}(R+1)}{2^{4} c^{4}(\sqrt{r}) \sin ^{4} \frac{\theta}{2}+1} .
\end{aligned}
$$

Now we finish the proof by deriving a contradiction. Since $\phi$ is $K$-quasiconformal,

$$
M(\Gamma) \leq K^{2} M(\phi \Gamma) .
$$


Combining Lemma 2.1, (52), and (53), we have

$$
\frac{1}{256}(R-r)\left(\frac{\pi}{2}-2 \arctan \frac{1}{\sqrt{r-1}}\right) \leq \frac{\pi c_{0}^{2} K^{2}(R+1)}{2^{4} c^{4}(\sqrt{r}) \sin ^{4} \frac{\theta}{2}+1} .
$$

Because the square integers $r<R$ are arbitrary, we take $R=4 r$. Lemma 3.2 implies that $c(\sqrt{r}) \rightarrow \infty$ as $r \rightarrow \infty$, and hence we have a contradiction.

For a positive real number $n \in \mathbb{R}_{+}-\{1\}$, we will denote simply by $n$ the real dilation $(z, t) \mapsto\left(n z, n^{2} t\right)$ We will use the following normalization repeatedly:

$$
\begin{aligned}
(\sqrt{n})^{-1} A_{\theta}^{n}(\sqrt{n})(z, t) & =(\sqrt{n})^{-1} A_{\theta}^{n}(\sqrt{n} z, n t) \\
& =(\sqrt{n})^{-1}\left(e^{n i \theta} \sqrt{n} z, n t+n\right) \\
& =\left(e^{n i \theta} z, t+1\right)=A_{n \theta}(z, t), \\
n^{-1} T_{(r, s)} n(z, t) & =n^{-1} T_{(r, s)}\left(n z, n^{2} t\right) \\
& =n^{-1}\left(n z+r, n^{2} t+s+2 r n \operatorname{Im} \bar{z}\right) \\
& =\left(z+\frac{r}{n}, t+\frac{s}{n^{2}}+\frac{2 r}{n} \operatorname{Im} \bar{z}\right)=T_{\left(r / n, s / n^{2}\right)}(z, t),
\end{aligned}
$$

where $n \in \mathbb{Z}, A_{\theta}(z, t)=\left(e^{i \theta} z, t+1\right)$ for $\theta \in[0,2 \pi)$, and

$$
T_{(r, s)}(z, t)=(z+r, t+s+2 r \operatorname{Im} \bar{z})
$$

for $r, s \in \mathbb{R}$.

Corollary 3.4. A rational screw parabolic automorphism is not quasiconformally conjugate to an irrational screw parabolic automorphism.

Proof. Let $A_{\theta}$ be a rational screw parabolic automorphism and $A_{\vartheta}$ be an irrational screw parabolic automorphism of $\mathscr{H}$. Suppose, to the contrary, that a $K$ quasiconformal map $\phi: \mathscr{H} \rightarrow \mathscr{H}$ exists such that $\phi \circ A_{\vartheta} \circ \phi^{-1}=A_{\theta}$. Then for any integer $n$,

$$
\phi \circ A_{\vartheta}^{n} \circ \phi^{-1}=A_{\theta}^{n} .
$$

Because $A_{\theta}$ is a rational screw parabolic automorphism, $A_{\theta}^{n_{0}}=T_{\left(0, n_{0}\right)}$ for some integer $n_{0}$. We conjugate both sides of (57) by a real dilation $\sqrt{n_{0}}$ and use (55) and (56) as follows:

$$
\begin{gathered}
\left(\sqrt{n_{0}}\right)^{-1} \phi A_{\vartheta}^{n_{0}} \phi^{-1} \sqrt{n_{0}}=\left(\sqrt{n_{0}}\right)^{-1} T_{\left(0, n_{0}\right)} \sqrt{n_{0}}, \\
\left(\sqrt{n_{0}}\right)^{-1} \phi\left(\sqrt{n_{0}} A_{n_{0} \vartheta}\left(\sqrt{n_{0}}\right)^{-1}\right) \phi^{-1} \sqrt{n_{0}}=T_{(0,1)} .
\end{gathered}
$$

This implies that a screw parabolic $A_{n_{0} \vartheta}(z, t)=\left(e^{n_{0} \vartheta} i z, t+1\right)$ is conjugate to a vertical translation $T_{(0,1)}$ by a quasiconformal mapping $\left(\sqrt{n_{0}}\right)^{-1} \phi \sqrt{n_{0}}$, which is a contradiction to Theorem 3.3. 
Applying the same idea as above, we also have:

Corollary 3.5. If two rational screw parabolic automorphisms are quasiconformally conjugate, then they have the same order.

Proposition 3.6. Let $A_{\theta}$ and $A_{\vartheta}$ be two distinct irrational screw parabolic automorphisms for $\theta, \vartheta \in(0,2 \pi)$. Then $A_{\theta}$ and $A_{\vartheta}$ are not quasiconformally conjugate to each other.

Proof. Using the normalization of (55), the proof follows the same idea of Proposition 4.15 of [Kim 2011].

We need the following theorem to prove that a screw parabolic automorphism is not quasiconformally conjugate to a horizontal translation.

Theorem F [Korányi and Reimann 1995]. If $\left\{\varphi_{n}: G \rightarrow \hat{\mathscr{H}}\right\}$, for a proper subset $G \subset \mathcal{H}$, is a sequence of $K$-quasiconformal mappings such that every mapping $\varphi_{n}$ omits two points $a_{n}$ and $b_{n}$ (depending on $\varphi_{n}$ ) with a distance at least $l$ ( $l$ a fixed positive number independent of $\varphi_{n}$ ), then there exists a locally uniformly convergent subsequence converging to a $K$-quasiconformal mapping or to a constant.

Theorem 3.7. Let $T_{(1,0)}(z, t)=(z+1, t+2 \operatorname{Im} \bar{z})$ be a horizontal translation and $A_{\theta}(z, t)=\left(e^{i \theta} z, t+1\right)$, for $\theta \in(0,2 \pi)$, be a screw parabolic automorphism of the Heisenberg group $\mathcal{H}$. Then $A_{\theta}$ is not quasiconformally conjugate to $T_{(1,0)}$.

Proof. Suppose, to the contrary, that a $K$-quasiconformal map $\phi: \mathscr{H} \rightarrow \mathscr{H}$ exists such that

$$
\phi \circ A_{\theta} \circ \phi^{-1}=T_{(1,0)} .
$$

Then for any integer $n$, we also have

$$
\phi \circ A_{\theta}^{n} \circ \phi^{-1}=T_{(1,0)}^{n}=T_{(n, 0)} .
$$

First consider the case that $A_{\theta}$ is a rational parabolic automorphism. Then there is a positive integer $n_{0}$ such that $A_{\theta}^{n_{0}}=T_{\left(0, n_{0}\right)}$. We conjugate both sides of (60) by a real dilation $n$ as follows:

$$
n^{-1}\left(\phi A_{\theta}^{n} \phi^{-1}\right) n=n^{-1} T_{(n, 0)} n=T_{(1,0)} .
$$

In particular, when $n=n_{0}$,

$$
n_{0}^{-1} \phi T_{\left(0, n_{0}\right)} \phi^{-1} n_{0}=T_{(1,0)} .
$$

Using that $T_{\left(0, n_{0}\right)}=\sqrt{n_{0}} T_{(0,1)}\left(\sqrt{n_{0}}\right)^{-1}$, we rewrite the left-hand side of (62) as

$$
\left(n_{0}^{-1} \phi \sqrt{n_{0}}\right) T_{(0,1)}\left(\left(\sqrt{n_{0}}\right)^{-1} \phi^{-1} n_{0}\right)=T_{(1,0)} \text {. }
$$

Because $\left(n_{0}\right)^{-1} \phi \sqrt{n_{0}}$ is also a $K$-quasiconformal mapping, (63) implies that the vertical translation $T_{(0,1)}$ is conjugate to the horizontal translation $T_{(1,0)}$ by the 
quasiconformal mapping $n_{0}^{-1} \phi \sqrt{n_{0}}$. This is a contradiction to Theorem 5.1 of [Miner 1994].

The second case is that $A_{\theta}$ is an irrational screw parabolic automorphism. Here we use the property that, under a mild condition, an infinite sequence of $K$-quasiconformal mappings is a normal family; see Theorem $\mathrm{F}$.

It is possible that the quasiconformal mapping $\phi$ of (59) does not fix the origin $(0,0)$. Hence, we conjugate both sides of (59) by a Heisenberg translation $m$ which sends $\phi(0,0)$ to $(0,0)$ ( $m$ might be the identity map) so that we have

$$
m \phi A_{\theta} \phi^{-1} m^{-1}=m \circ T_{(1,0)} \circ m^{-1} .
$$

If $m \circ T_{(1,0)} \circ m^{-1}$ is a vertical translation, then we have proved the theorem. Otherwise, $m T_{(1,0)} m^{-1}$ is a nonvertical translation. Now we conjugate (64) by a rotation $\lambda:(z, t) \mapsto(\lambda z, t)$ for a unit $\lambda \in \mathbb{C}$ so that $\lambda m T_{(1,0)} m^{-1} \lambda=T_{(r, s)}$ for some real numbers $r \neq 0$ and $s$ :

$$
\lambda m \phi A_{\theta} \phi^{-1} m^{-1} \lambda^{-1}=\lambda m T_{(1,0)} m^{-1} \lambda^{-1}=T_{(r, s)} .
$$

Let $\varphi=\lambda m \phi$. Then $\varphi$ is a $K$-quasiconformal mapping, fixes the origin $(0,0)$ and

$$
\varphi \circ A_{\theta} \circ \varphi^{-1}=T_{(r, s)} .
$$

(We note that if $\phi$ fixes $(0,0)$, then $m$ and $\lambda$ are the identity map, $T_{(r, s)}=T_{(1,0)}$, and $\varphi=\phi$.)

Let $n$ be any integer; then from (66), we have

$$
\varphi A_{\theta}^{n} \varphi^{-1}=T_{(r, s)}^{n}=T_{(n r, n s)}
$$

because $r$ and $s$ are real numbers. Evaluating (67) at $(0,0)$ shows that

$$
\varphi(0, n)=(n r, n s) .
$$

We conjugate both sides of (67) by a real dilation $n$ and use equations (55) and (56) as follows:

$$
\begin{gathered}
n^{-1} \varphi A_{\theta}^{n} \varphi^{-1} n=n^{-1} T_{(n r, n s)} n, \\
n^{-1} \varphi\left(\sqrt{n} A_{n \theta}(\sqrt{n})^{-1}\right) \varphi^{-1} n=T_{(r, s / n)} .
\end{gathered}
$$

Because $A_{\theta}$ is an irrational screw parabolic, there is a subsequence $\left\{A_{n_{k} \theta}: k \in \mathbb{N}\right\}$ which converges to the vertical translation $T_{(0,1)}$. For each $k \in \mathbb{N}$, let $\psi_{k}=n_{k}^{-1} \varphi \sqrt{n_{k}}$. Then each $\psi_{k}$ is again $K$-quasiconformal, fixes $(0,0)$, and

$$
\psi_{k} A_{n_{k} \theta} \psi_{k}^{-1}=T_{\left(r, s / n_{k}\right)} .
$$

To apply Theorem F, let $G=\mathscr{H}-\{(0,0)\}$ and restrict each $\psi_{k}$ on $G$. Thus, we have an infinite sequence of $K$-quasiconformal mappings, $\mathscr{F}_{\mathcal{F}}=\left\{\psi_{k}: G \rightarrow \hat{\mathscr{H}} \mid k \in \mathbb{N}\right\}$. Note 
that each $\left.\psi_{k}\right|_{G}$ omits $(0,0)$ and $\infty$ in $\hat{\mathscr{H}}$. Hence, the sequence $\mathscr{F}$ has a convergent subsequence whose limit, say $\psi$, is a $K$-quasiconformal homeomorphism for the following reason: for any integer $m$,

$$
\psi_{k}(0, m)=n_{k}^{-1} \varphi \sqrt{n_{k}}(0, m)=\left(m r, \frac{m s}{n_{k}}\right)
$$

converges to $(m r, 0)$ as $k \rightarrow \infty$. Thus, $\psi(0, m)=(m r, 0)$ for any integer $m$, and hence $\psi$ is not a constant function. We now extend $\psi$ to $\mathscr{H}$ by defining $\psi(0,0)=(0,0)$. From $(70)$, we have $\psi \circ T_{(0,1)} \circ \psi^{-1}=T_{(r, 0)}$ which is a contradiction by Theorem 5.1 of [Miner 1994].

Corollary 3.8. Let $T_{(1,0)}(z, t)=(z+1, t+2 \operatorname{Im} \bar{z})$ be a horizontal translation and $A(z, t)=\left(e^{i \theta} z, t+1\right)$, for $\theta \in(0,2 \pi)$, be a screw parabolic automorphism in the Heisenberg group Het $\Gamma_{1}$ and $\Gamma_{2}$ be the cyclic groups generated by $T_{(1,0)}$ and $A$, respectively. Then there exists no quasiconformal mapping between $\mathscr{H} / \Gamma_{1}$ and $\mathcal{H} / \Gamma_{2}$. In particular, $\Gamma_{1}$ is not quasiconformally conjugate to $\Gamma_{2}$.

\section{Acknowledgements}

The author is grateful to Ara Basmajian, Elisha Falbel and Ioannis Platis for many helpful comments and discussions and to the University of Berne for their hospitality. The author also thanks to the referee of this paper for pointing out some inaccuracies in the first draft of the manuscript.

\section{References}

[Basmajian and Miner 1998] A. Basmajian and R. Miner, "Discrete subgroups of complex hyperbolic motions", Invent. Math. 131:1 (1998), 85-136. MR 99e:32039 Zbl 0901.32018

[Bers 1970] L. Bers, "On boundaries of Teichmüller spaces and on Kleinian groups, I", Ann. of Math. (2) 91 (1970), 570-600. MR 45 \#7044 Zbl 0197.06001

[Goldman 1999] W. M. Goldman, Complex hyperbolic geometry, Oxford University Press, New York, 1999. MR 2000g:32029 Zbl 0939.32024

[Heinonen and Holopainen 1997] J. Heinonen and I. Holopainen, "Quasiregular maps on Carnot groups”, J. Geom. Anal. 7:1 (1997), 109-148. MR 99i:30037 Zbl 0905.30018

[Izeki 2000] H. Izeki, "Quasiconformal stability of Kleinian groups and an embedding of a space of flat conformal structures”, Conform. Geom. Dyn. 4 (2000), 108-119. MR 2002a:57056 Zbl 0959. 58027

[Kapovich 2008] M. Kapovich, "Kleinian groups in higher dimensions", pp. 487-564 in Geometry and dynamics of groups and spaces (Bonn, 2006), edited by M. Kapranov et al., Progr. Math. 265, Birkhäuser, Basel, 2008. MR 2009g:30043 Zbl 1147.30028

[Kim 2011] Y. Kim, "Quasiconformal stability for isometry groups in hyperbolic 4-space", Bull. Lond. Math. Soc. 43:1 (2011), 175-187. MR 2012b:57036 Zbl 1218.57013

[Korányi and Reimann 1985] A. Korányi and H. M. Reimann, "Quasiconformal mappings on the Heisenberg group”, Invent. Math. 80:2 (1985), 309-338. MR 86m:32035 Zbl 0567.30017 
[Korányi and Reimann 1995] A. Korányi and H. M. Reimann, "Foundations for the theory of quasiconformal mappings on the Heisenberg group", Adv. Math. 111:1 (1995), 1-87. MR 96c:30021 Zbl 0876.30019

[Marden 1974] A. Marden, "The geometry of finitely generated Kleinian groups”, Ann. of Math. (2) 99 (1974), 383-462. MR 50 \#2485 Zbl 0282.30014

[Maskit 1988] B. Maskit, Kleinian groups, Grundlehren der Mathematischen Wissenschaften 287, Springer, Berlin, 1988. MR 90a:30132 Zbl 0627.30039

[Miner 1994] R. R. Miner, "Quasiconformal equivalence of spherical CR manifolds", Ann. Acad. Sci. Fenn. Ser. A I Math. 19:1 (1994), 83-93. MR 95b:30032 Zbl 0797.30016

[Parker and Platis 2010] J. R. Parker and I. D. Platis, "Complex hyperbolic quasi-Fuchsian groups", pp. 309-355 in Geometry of Riemann surfaces (Anogia, 2007), edited by F. P. Gardiner et al., London Math. Soc. Lecture Note Ser. 368, Cambridge University Press, 2010. MR 2011k:30051 Zbl 1204.57015

[Schwartz 2007] R. E. Schwartz, Spherical CR geometry and Dehn surgery, Annals of Mathematics Studies 165, Princeton University Press, 2007. MR 2009a:32047 Zbl 1116.57016

Received March 27, 2013. Revised November 24, 2013.

YOUNGJU KIM

DEPARTMENT OF MATHEMATICS

Korea InSTITUTE FOR AdVANCED STUdy

HoEgiro 85 DONGDAEMUN-GU

SEOUL 130-722

SOUTH KOREA

geometer1@kias.re.kr 


\title{
PACIFIC JOURNAL OF MATHEMATICS
}

\author{
msp.org/pjm
}

Founded in 1951 by E. F. Beckenbach (1906-1982) and F. Wolf (1904-1989)

\section{EDITORS}

Don Blasius (Managing Editor)

Department of Mathematics

University of California

Los Angeles, CA 90095-1555

blasius@math.ucla.edu

\author{
Paul Balmer \\ Department of Mathematics \\ University of California \\ Los Angeles, CA 90095-1555 \\ balmer@math.ucla.edu \\ Robert Finn \\ Department of Mathematics \\ Stanford University \\ Stanford, CA 94305-2125 \\ finn@math.stanford.edu \\ Sorin Popa \\ Department of Mathematics \\ University of California \\ Los Angeles, CA 90095-1555 \\ popa@math.ucla.edu
}

\author{
Vyjayanthi Chari \\ Department of Mathematics \\ University of California \\ Riverside, CA 92521-0135 \\ chari@math.ucr.edu \\ Kefeng Liu \\ Department of Mathematics \\ University of California \\ Los Angeles, CA 90095-1555 \\ liu@math.ucla.edu \\ Jie Qing \\ Department of Mathematics \\ University of California \\ Santa Cruz, CA 95064 \\ qing@ cats.ucsc.edu
}

\section{PRODUCTION}

Silvio Levy, Scientific Editor, production@msp.org

\section{SUPPORTING INSTITUTIONS}

ACADEMIA SINICA, TAIPEI

CALIFORNIA INST. OF TECHNOLOGY

INST. DE MATEMÁTICA PURA E APLICADA

KEIO UNIVERSITY

MATH. SCIENCES RESEARCH INSTITUTE

NEW MEXICO STATE UNIV.

OREGON STATE UNIV.

\author{
STANFORD UNIVERSITY \\ UNIV. OF BRITISH COLUMBIA \\ UNIV. OF CALIFORNIA, BERKELEY \\ UNIV. OF CALIFORNIA, DAVIS \\ UNIV. OF CALIFORNIA, LOS ANGELES \\ UNIV. OF CALIFORNIA, RIVERSIDE \\ UNIV. OF CALIFORNIA, SAN DIEGO \\ UNIV. OF CALIF., SANTA BARBARA
}

\author{
Daryl Cooper \\ Department of Mathematics \\ University of California \\ Santa Barbara, CA 93106-3080 \\ cooper@math.ucsb.edu \\ Jiang-Hua Lu \\ Department of Mathematics \\ The University of Hong Kong \\ Pokfulam Rd., Hong Kong \\ jhlu@maths.hku.hk \\ Paul Yang \\ Department of Mathematics \\ Princeton University \\ Princeton NJ 08544-1000 \\ yang@math.princeton.edu
}

These supporting institutions contribute to the cost of publication of this Journal, but they are not owners or publishers and have no responsibility for its contents or policies.

See inside back cover or msp.org/pjm for submission instructions.

The subscription price for 2014 is US $\$ 410 /$ year for the electronic version, and \$535/year for print and electronic.

Subscriptions, requests for back issues and changes of subscribers address should be sent to Pacific Journal of Mathematics, P.O. Box 4163, Berkeley, CA 94704-0163, U.S.A. The Pacific Journal of Mathematics is indexed by Mathematical Reviews, Zentralblatt MATH, PASCAL CNRS Index, Referativnyi Zhurnal, Current Mathematical Publications and Web of Knowledge (Science Citation Index).

The Pacific Journal of Mathematics (ISSN 0030-8730) at the University of California, c/o Department of Mathematics, 798 Evans Hall \#3840, Berkeley, CA 94720-3840, is published twelve times a year. Periodical rate postage paid at Berkeley, CA 94704, and additional mailing offices. POSTMASTER: send address changes to Pacific Journal of Mathematics, P.O. Box 4163, Berkeley, CA 94704-0163.

PJM peer review and production are managed by EditFLOW ${ }^{\circledR}$ from Mathematical Sciences Publishers.

\section{PUBLISHED BY}

\section{mathematical sciences publishers \\ nonprofit scientific publishing}

http://msp.org/

(C) 2014 Mathematical Sciences Publishers 


\section{PACIFIC JOURNAL OF MATHEMATICS}

Volume $270 \quad$ No. $1 \quad$ July 2014

Hermitian categories, extension of scalars and systems of sesquilinear forms

EVA BAYER-FLUCKIGER, URIYA A. FIRST and DANIEL A.

MOLDOVAN

Realizations of the three-point Lie algebra $\mathfrak{s l}(2, \mathscr{R}) \oplus\left(\Omega_{\mathscr{R}} / d \mathscr{R}\right)$

BEN COX and ELIZABETH JURISICH

Multi-bump bound state solutions for the quasilinear Schrödinger equation with critical frequency

YUXIA GUO and ZHONGWEI TANG

On stable solutions of the biharmonic problem with polynomial growth

Hatem Hajlaoui, Abdellaziz Harrabi and Dong Ye

Valuative multiplier ideals

ZHENGYU HU

Quasiconformal conjugacy classes of parabolic isometries of complex hyperbolic space

YOUNGJU KIM

On the distributional Hessian of the distance function

Carlo MantegazZa, Giovanni Mascellani and Gennady URALTSEV

Noether's problem for abelian extensions of cyclic $p$-groups

IVO M. MICHAILOV

Legendrian $\theta$-graphs

Danielle O’Donnol and Elena PaVelescu

A class of Neumann problems arising in conformal geometry

WEIMIN SHENG and LI-XIA YUAN

Ryshkov domains of reductive algebraic groups

TAKAO WATANABE 